\title{
THE RELATIONSHIP BETWEEN CENTRAL NUCLEAR SCATTER AND PERINUCLEAR RETRODOTS IN THE HUMAN CRYSTALLINE LENS
}

\author{
G. ADRIEN SHUN-SHIN, ANTHONY J. BRON, NICHOLAS P. BROWN, JOHN M. SPARROW \\ Oxford
}

\begin{abstract}
SUMMARY
Retrodots are common perinuclear features of agerelated cataract. Clinical observation suggested that retrodots were associated with increased nuclear scatter. To test this hypothesis, retrospective data were analysed from 78 non-diabetic subjects (118 eyes), of at least 50 years of age, who had neither subscapsular nor axial spoke opacities. Retrodots were graded using the Oxford Clinical Cataract Classification and Grading system and central nuclear scatter was assessed by image analysis of Scheimpflug photographic negatives. Regression analysis in groups was performed according to the "intra-class correlation model" either considering retrodots as a graded feature (grades 0-5) or as a dichotomous variable (present or absent). After adjusting for age (treated as a co-variate), a highly significant association $\left(P<10^{-6}\right)$ was found between retrodots and increased nuclear scatter in each analysis. It is suggested that the co-existence of these two cataract types may reflect a common cataractogenic mechanism, possibly oxidative stress.
\end{abstract}

Lens retrodots are small disc-shaped features seen in the perinuclear cortex. ${ }^{1}$ When seen in retro-illumination they are rounded features (Fig. 1) and show reversal of background illumination. ${ }^{2}$ On specular microscopy, they appear dome shaped. They are approximately $300 \mu$ in diameter.

Nuclear changes are a common feature of age-related cataract. In the NHANES study, they occurred in $10 \%$ of the subjects aged between 45 to 74 years. ${ }^{3}$ On biomicroscopy, the features of nuclear cataract include white nuclear scatter and nuclear brunescense. ${ }^{4,5}$ The terms nuclear opalescence and nuclear colour have also been used. ${ }^{6}$ More complex descriptions of nuclear changes are at present being developed. ${ }^{7}$

Brown Scheimpflug slit-lamp photography ${ }^{8}$ combined

Correspondence to: G. Adrien Shun-Shin, Clinical Cataract Research Unit, Nuffield Laboratory of Ophthalmology, Walton Street, Oxford OX2 6AW. with the measurement of the density of the photographic negatives, gives an objective measure of nuclear scatter. ${ }^{9}$ Central nuclear scatter has been shown to have a high correlation with age in a normal control population $\left(\mathrm{R}^{2}=\right.$ $\left.0.82, \mathrm{P}=10^{-6}\right) .{ }^{10}$ Nuclear brunescense is associated with a spectrally selective absorption of light. The densitometric method of measuring nuclear scatter does not allow for this factor so that the intensity of light scatter by the nucleus will be underestimated in the presence of brunescence.

Clinical observation suggested to us that the presence of retrodots was associated with an increase in nuclear changes. In this retrospective study we investigate this possible relationship.

\section{MATERIALS AND METHODS}

\section{Selection of patients:}

This was a retrospective survey of the records of patients who had been examined by one observer (GASS) in the Clinical Cataract Research Unit over a two-year period. Patients aged 50 years or more, with age related cataracts

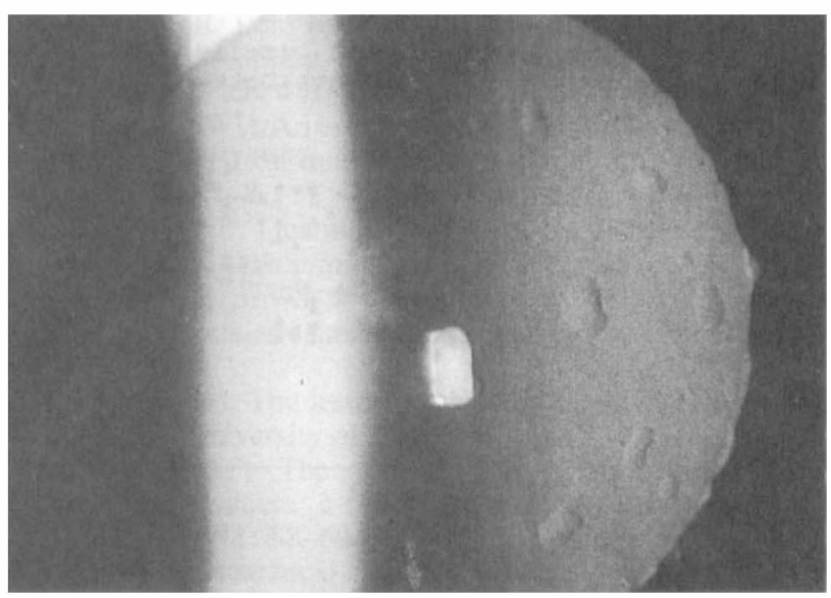

Fig. 1. Lens retrodots seen in retroillumination. 


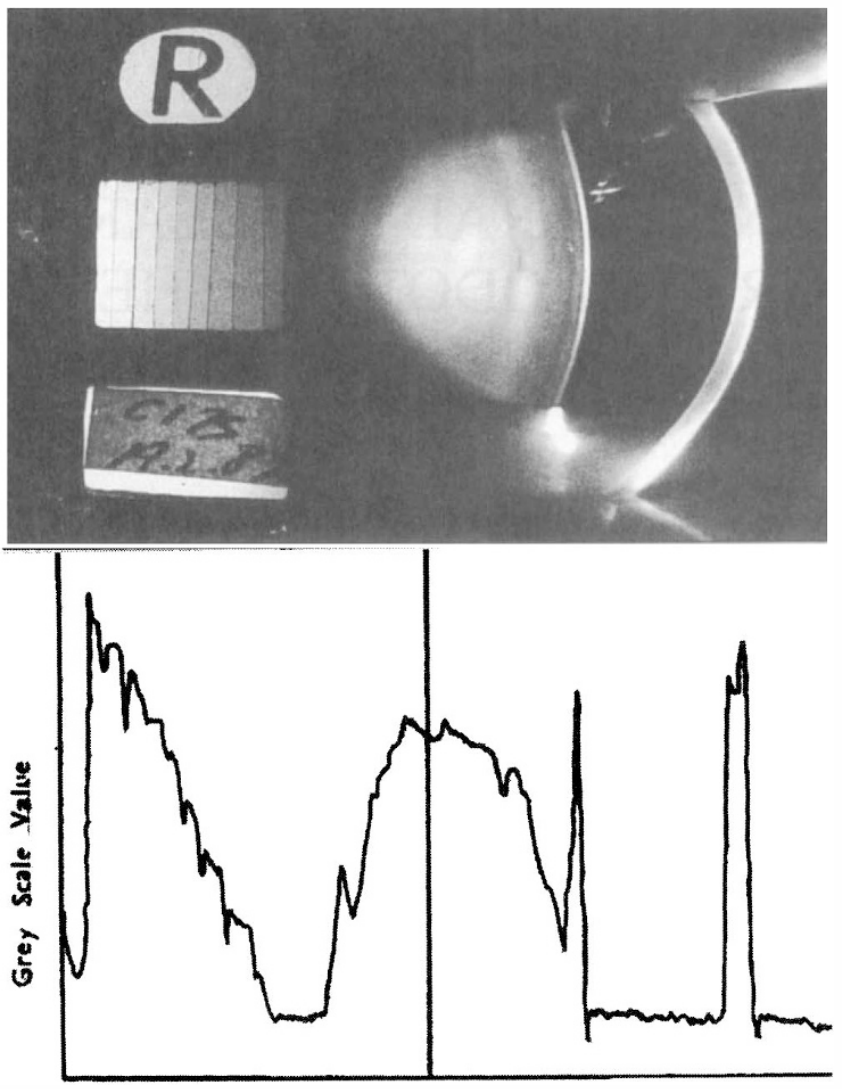

$Y=17.0 N^{\prime} s$

Fig. 2 (a) Scheimpflug slit-image photograph of a lens with retrodots and nuclear scatter. Arrow points to a retrodot in perinuclear cortex. On the left is the neutral density scale. (b) An axial density scan of the lens in Fig. $2 a$. On the ordinate is the neutral density value in arbitrary units. The cursor line is just to the left of the nuclear dip. The density at the cursor is 17.0 neutral densities (in arbitrary units).

were identified. All posterior subcapsular cataracts (PSC) were excluded because the PSC can scatter light forward and thus artefactually affect the measurement of nuclear

CENTRAL NUCLEAR SCATTER UE AGE

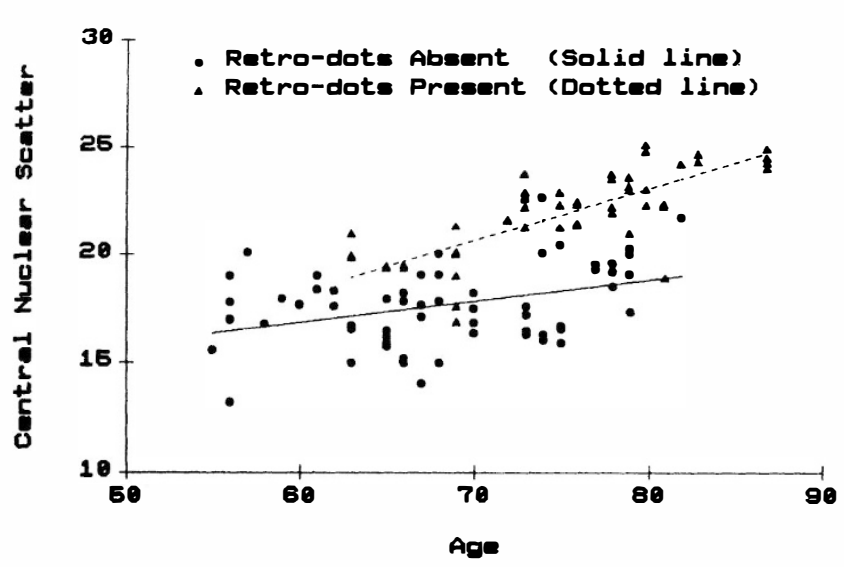

Fig. 3. Graph of central nuclear scatter (arbitary units) and age of patient. The circles represent lenses with no retrodots and the triangles, lenses with retrodots. Regression lines for the two groups are shown. scatter. Eyes with spoke opacities were excluded if the spoke opacities involved the central $5 \mathrm{~mm}$ of the lens. Spoke opacities scatter light forward and cast shadows posteriorly and can thus affect the measurement of nuclear scatter.

Out of a total of 203 records examined, 78 patients with at least one suitable eye were identified. Twelve eyes were aphakic and 26 eyes were rejected because of PSC or spoke opacity. A total of 118 eyes thus fitted the inclusion criteria of the study.

\section{METHOD}

Lenses were examined after full pupillary dilatation to at least $7 \mathrm{~mm}$ diameter using G. Phenylephrine $10 \%$ and G. Tropicamide $1 \%$ repeated at least twice. The lenses were graded using the Oxford Clinical Cataract Grading System. ${ }^{4}$ In this system, retrodots are graded on a 0 to 5 scale according to numbers present. Lenses were then photographed using the Brown Scheimpflug slit image camera $^{8}$ and the Oxford retro-illumination camera. ${ }^{11}$

The Scheimpflug slit-image photographic negatives were analysed on the Oxford Modular Cataract Image Analysis System (MCIAS). ${ }^{9}$ An axial density scan was performed of the Scheimpflug negative and the density of the central nuclear dip was measured relative to neutral density standards incorporated into the Brown Scheimpflug camera. Figure 2a shows a Scheimpflug picture of a nuclear cataract and Figure $2 \mathrm{~b}$ shows the axial density scan with the central nuclear dip.

The repeatability of the measurement of the density at the central nuclear dip has been investigated. There is a 0.95 probability that any individual measurement error will lie within the range \pm 0.43 neutral density units which corresponds to $\pm 4 \%$ of the dynamic range of the measurement. ${ }^{9}$

The general statistical method employed was that described by Rosner, for use with continuous dependent variables in ophthalmology where two eyes are involved in the analysis. ${ }^{12,13}$ Rosner's "intraclass correlation model" is an efficient statistical technique in studies with a two eye design such as this, and avoids over-estimation of significance levels associated with the high inter-eye correlation found in ophthalmological data. ${ }^{12,13,14}$ The poten-

Table I. Analysis of variance using interclass correlation model

\begin{tabular}{lcccrcc}
\hline \multicolumn{7}{c}{ Retro-Dots Clinical Grading Scale } \\
Source & SSq & DF & MS & F & P \\
\hline Age & 427.2 & 1 & 427.2 & 111 & $<10-6$ \\
Retro-dots (GR) & 250.6 & 4 & 62.65 & 16 & $<10-6$ \\
Patients & 257.8 & 67 & 3.8478 & 1 & \\
Eyes & 28.1 & 45 & 0.6244 & & \\
Total & 963.7 & 117 & & & \\
\hline
\end{tabular}

$\left(\mathrm{R}^{2}\right.$ for the model $\left.=0.70, \mathrm{R}=0.83\right)$.

$\mathrm{SSq}=$ sum of squares

$\mathrm{DF}=$ degrees of freedom

MS $=$ mean square

$\mathrm{F}=$ variance ratio

$\mathrm{P}=$ probability

On average lenses with retro-dots scatter by 2.8 units more than those without 
Table II. Analysis of variance using interclass correlation model

\begin{tabular}{lccccc}
\hline \multicolumn{7}{c}{ Retro-Dots, Dichotomous Scale } & & \\
Source & SSq & DF & MS & F & P \\
\hline Age & 427.2 & 1 & 427.2 & 102 & $<10-6$ \\
Retro-dots & 188.1 & 1 & 188.1 & 45 & $<10-6$ \\
Age retro-dots & 40.1 & 1 & 40.1 & 9.6 & 0.0028 \\
Patients & 278.8 & 67 & 4.16 & 1 & \\
Eyes & 29.5 & 47 & 0.62 & & \\
Total & 963.7 & 117 & & & \\
\hline
\end{tabular}

$\left(\mathrm{R}^{2}\right.$ for the model $\left.=0.68, \mathrm{R}=0.82\right)$.

$\mathrm{SSq}=$ sum of squares

$\mathrm{DF}=$ degrees of freedom

$\mathrm{MS}=$ mean square

$\mathrm{F}=$ variance ratio

$\mathrm{P}=$ probability

On average lenses with retro-dots scatter by 2.8 units more than those without

tially confounding effect of age was accounted for prior to fitting other terms of interest in the multivariate model. The progam used for statistical analysis was the Generalized Linear Interactive Modelling System (GLIM) of the Numerical Algorithm Group.

\section{RESULTS}

Figure 3 shows a plot of the central nuclear scatter against the age of the patient. Both eyes are plotted. Circles represent lenses without retrodots and triangles lenses with retrodots. From the regression lines, it is evident that central nuclear scatter in lenses with retrodots is greater than in those without.

In the first statistical analysis, the effect of the grade of retrodots (grade 0 to 5) was examined. In this multiple regression analysis, the grade of retrodots was significantly associated with central nuclear scatter after accounting for age (See Table I "Retrodots-clinical grading scale"). This effect was such that increased retrodots were associated with increased central nuclear scatter.

In the second analysis, the grading scale was collapsed and retrodots were regarded as either present or absent (See Table II. "Retrodots-dichotomous scale"). The presence of retrodots was clearly associated with increased central nuclear scatter. The significant interaction term (Age. Retrodots) indicated that the slopes for the age $v s$ scatter regression lines were different for the two groups as illustrated in Figure 3.

Holding the age slopes parallel for the two groups showed that on average the central nuclear scatter was 2.8 neutral density units greater for lenses with retrodots compared to those without $\left(\mathrm{P}<10^{-6}\right)$.

\section{DISCUSSION}

There is a statistically significant relationship between the presence and grade of retrodots and central nuclear scatter as measured by image analysis of Scheimpflug photographic negatives. The aetiology of these features is uncertain. Retrodots are thought to contain calcium oxalate $^{15}$ and it has been postulated that oxalate is the result of oxidation of ascorbate. ${ }^{1}$ Benedek has suggested that lens protein aggregates of molecular weight greater than
$50 \times 10^{6}$ will cause light scatter. ${ }^{16}$ The high molecular weight aggregates occur mainly in the lens nucleus and have been correlated in vitro by Spector with increases in light scatter. ${ }^{17}$ Harding has postulated that nuclear cataract results in part from post-translational modification of long lived lens crystallins. This alters surface charge and allows unfolding of the crystallin tertiary structure. The unfolded crystallins display reactive thiol groups and form disulphide bonds. This results in disulphide-bonded macromolecular aggregates capable of light scatter. The causes of crosslinking are probably multifactorial and include oxidative stress in the lens. ${ }^{18}$ However, whether or not oxidative stress is important the two types of cataract appear to share a common aetiology.

\section{CONCLUSIONS}

In this study a significant association is demonstrated between two morphologically distinct lens features i.e. perinuclear retrodots and central nuclear scatter. It is suggested that retrodots and nuclear scatter may act as markers for oxidative stress in the lens. The evolution of retrodots and nuclear scatter in ongoing trials of antioxidant drugs in the medical treatment of cataract will thus be of great interest.

Key words: Lens retrodots, nuclear cataract, scheimpflug photography, image analysis.

\section{REFERENCES}

1. Bron AJ and Brown NAP: Perinuclear lens retrodots: a role for ascorbate in cataractogenesis. B J Ophthalmol 1987, 71: 86-95.

2. Brown NAP: The visibility of transparent objects in the eye by retro-illumination. B J Ophthalmol 1971, 55: 517-24.

3. Hiller R, Sperduto RD, Ederer F: Epidemiologic associations with nuclear, cortical and posterior subcapsular cataract. Am J Epidemiol 1986, 124: 916-25.

4. Sparrow JM, Bron AJ, Brown NAP, Hill AR, Ayliffe W: The Oxford clinical cataract classification and grading system. Int Ophthalmol 1986; 9: 207-25.

5. Sparrow JM, Ayliffe W, Bron AJ, Brown NAP, Hill AR: Inter-Obseryer and intra-observer variability of the Oxford clinical cataract classification and grading system. Int Ophthalmol 1988, 11: 151-7.

6. Chylack LT, Leske C, McCarthy D, Khu P, Kashiwati T, Sperduto R: Lens opacities classification system II (LOCS II). Arch Ophthalmol 1989, 107: 991-7.

7. Getty DJ, Pickett RM, Chylack LT, Leske C, McCarthy D, Huggins AWF: An enriched set of features of nuclear cataract identified by multidimensional scaling. Curr Eye Res 1989, 8/1: 1-8.

8. Brown NAP: Quantitative slit-image photography of the lens. Trans Ophthalmol Soc UK 1972, 92: 303-17.

9. Sparrow J, Brown NAP, Bron AJ, Shun-Shin GA: The Oxford modular cataract image analysis system. Eye 1990, 4: $638-48$.

10. Sparrow JM: The lens in diabetes. D. Phil Thesis, Linacre College, University of Oxford. 1988.

11. Brown NAP: The Oxford Retro-illumination cataract recording camera: a new instrument. $J$ Audiovis Media in Med 1988, 11: 58-60.

12. Rosner B: Statistical methods in ophthalmology: an adjustment for the intra-correlation between eyes. Biometrics 1982, 38: 105-14.

13. Ederer F: Shall we count numbers of eyes or numbers of subjects? Arch Ophthalmol 1973, 89: 1-2. 
14. Ray WA, O'Day DM, Head WS et al: Statistical analysis for experimental models of ocular disease: continuous response measures. Curr. Eye Res 1985, 4: 585-97.

15. Harding CV, Chylack LT, Susan SR, Lo W-K, Brobowski WF: Calcium-containing opacities in the human lens. Invest Ophthalmol Vis Sci: 83, 1194-1202.

16. Benedek GB: Theory of the transparency of the lens. Applied Optics 1971, 10: 459-73.
17. Spector A, Li S, Sigelman J: Age-dependent changes in the molecular size of human lens proteins and their relationship to light scatter. Invest Ophthalmol Vis Sci 1974, 13: 795-801.

18. Harding $\mathrm{J}$ and Crabbe MJC: The lens: development, proteins, metabolism and cataract. In: Davson H. The Eye. Vol 1b. Vegetative physiology and biochemistry. London: Academic Press 1984, 207-492. 